\title{
Mujeres políticas y medios de comunicación: representación en prensa escrita del gobierno catalán $(2010)^{1}$
}

\author{
Núria FERNÁNDEZ GARCÍA \\ Universidad Autónoma de Barcelona (UAB) \\ nuria.fernandez@uab.cat
}

Recibido: 09/04/2012

Aceptado: 18/12/2012

\begin{abstract}
Resumen
Este artículo explora la representación en los medios del último gobierno catalán formado a partir de las elecciones de noviembre de 2010. Aplicando el análisis de contenido a una muestra de artículos extraída de cinco diarios españoles (El País, El Mundo, ABC, La Vanguardia, El Periódico de Catalunya), se analizan los primeros días de la formación del gobierno. Se compara la cobertura recibida por los miembros del govern utilizando seis variables: 1) visibilidad, 2) menciones a la apariencia, vida privada y referencias a la experiencia profesional, 3) menciones al género y asociación con una figura masculina, 4) nombre, 5) declaraciones, 6) características de la personalidad, y 7) tono de la cobertura. El análisis refleja cómo las mujeres políticas aún reciben una cobertura diferenciada de la de sus colegas varones.

Palabras clave: género, política, prensa, estereotipos, gobierno catalán

\section{Women Politicians and the Media: Catalan Government Representation in the Print Media (2010)}

\begin{abstract}
This article explores the media representation of the Catalan government elected in the elections held by November 2010. Using content analysis on a sample of stories from five Spanish newspapers (El País, El Mundo, ABC, La Vanguardia, El Periódico de Catalunya), the first days of the government formation will be analyzed. The article compares the coverage of the male and female members of the government using six indicators: 1) visibility, 2) mentions to the appearance, personal life and references to professional background, 3) mentions to the politician gender and link to a male mentor, 4) name, 5) quotations, 6) personal traits, and 7) tone of the coverage. The study shows how women politician are still disadvantaged in relation to their male counterparts.
\end{abstract}

Keywords: gender, politics, print media, stereotypes, Catalan government.

\section{Referencia normalizada}

FERNÁNDEZ GARCÍA, Núria (2013): "Mujeres políticas y medios de comunicación: representación en prensa escrita del gobierno catalán (2010)". Estudios sobre el mensaje periodístico. Vol. 19, Núm. 1, págs.: 365-381. Madrid, Servicio de Publicaciones de la Universidad Complutense.

Sumario: 1. Introducción. 2. Metodología. 3. Resultados; 3.1. Visibilidad; 3.2.Vida personal y profesional; 3.3. Género y figura masculina; 3.4. Primer nombre; 3.5. Declaraciones; 3.6. Características personales; 3.7. Tono de la cobertura. 4. Conclusiones. 5. Notas para el debate. 6. Referencias bibliográficas.

1 Este estudio ha recibido una subvención del Institut Català de les Dones (ICD), dentro de su programa de becas para incentivar la investigación en materia de estudios de género y de las mujeres para el periodo 2010 - 2011 


\section{Introducción}

Actualmente, la definición de la política como espacio masculino comienza a debilitarse con el aumento de la presencia de mujeres en altos cargos políticos. Brasil, Islandia o Alemania son sólo algunos de los países donde actualmente el máximo cargo ejecutivo es ocupado por una mujer. Además, la presencia de la mujer en las asambleas legislativas también ha aumentado significativamente en el mundo, ocupando España el octavo lugar de la Unión Europea en participación femenina en parlamentos nacionales (Inter-Parliamentary Union, 2011). No obstante, a pesar de este aumento en el número de mujeres que acceden a puestos políticos de alto nivel, son numerosos los estudios que muestran que la mujer política aún sigue siendo representada de forma estereotipada en los medios (Aday y Devitt, 2001; Braden, 1996; Falk, 2008; Fernández, 2008, 2010; Kahn, 1992, 1994, 1996; Kahn y Goldenberg, 1991; Kittilson y Fridkin, 2008; Trimble, 2007).

Por ello es necesario analizar cómo los medios representan a las mujeres políticas. Si las mujeres reciben un tratamiento diferenciado éste puede tener consecuencias en el proceso democrático (Scammell y Semetko, 2000). Y esto es así porque actualmente los medios tienen un gran impacto en las percepciones del público sobre los líderes políticos y en especial sobre las mujeres políticas (Sreberny y Van Zoonen, 2000). Por lo tanto, representar a las mujeres como excepción a la norma política puede llevar al público a ver a éstas como contendientes inusuales y menos viables (Banwart et al., 2003), disminuir las oportunidades de que la ciudadanía las acepte como potenciales representantes políticas e incluso no votarlas (Braden, 1996).

Pero, ¿cómo representan los medios a las mujeres que ostentan un cargo político?, ¿es el género de éstas el rasgo más importante en la cobertura, por delante de su competencia política?

La presencia de las mujeres en la vida política española ha sido, hasta 2004, escasa, lo que ha provocado que hubiese pocos casos de estudio. En 2004, con la llegada al poder de José Luís Rodríguez Zapatero (Partido Socialista Obrero Español, PSOE) se configura el primer gobierno paritario en la historia de España, con un reparto equitativo de ministerios entre hombres y mujeres, que contaría además con la primera mujer en asumir el cargo de la vicepresidencia del gobierno. En 2007 esta paridad pasa a ser norma, al aprobar el Gobierno español la Ley Orgánica 3/2007, de 22 de marzo, para la igualdad efectiva de hombres y mujeres, más conocida como Ley de Igualdad, que consagra el principio de presencia equilibrada de mujeres y hombres en las listas electorales y en los nombramientos realizados por los poderes públicos, entendiendo por composición equilibrada aquélla en la que el número de personas de cada uno de los sexos no sea superior al $60 \%$ ni inferior al $40 \%$. No obstante, tras las elecciones generales de noviembre de 2011 el gobierno presidido por Mariano Rajoy (Partido Popular, PP) contará sólo con cuatro ministras de un total de 13 carteras ministeriales, lo que representa una presencia femenina en el Consejo de Ministros del $30.76 \%$.

En lo que concierne a las asambleas legislativas, tras las elecciones de 2011, el porcentaje de mujeres en el Congreso se mantiene respecto a la anterior legislatura, con un $36.3 \%$ de diputadas, y en el Senado aumenta ligeramente la presencia de mujeres, alcanzando el 35.6\%. Ambos porcentajes se encuentran por debajo del mínimo establecido por la Ley de Igualdad. 
Más equilibrada resulta la participación femenina en las asambleas legislativas de las comunidades autónomas. Tras las celebración de las elecciones autonómicas catalanas en 2010, el porcentaje de mujeres en el conjunto de parlamentos autonómicos se eleva al 42.2\% (datos extraídos del Instituto Nacional de Estadística (INE, 2011). En el caso de Cataluña, las últimas elecciones incrementan el porcentaje de participación femenina en el Parlament, pasando del $36.3 \%$ en la anterior legislatura, al $41.5 \%$ en la legislatura actual.

En cuanto a la composición del Govern catalán, ninguna mujer ha asumido el máximo cargo ejecutivo: la presidencia de la Generalitat y, en los últimos años, la presencia femenina en el ejecutivo ha sido discreta. En 2003 durante el primer gobierno tripartito (integrado por una coalición de partidos de izquierda), presidido por el socialista Pasqual Maragall, la presencia femenina en los diferentes departamentos del gobierno es de un $25 \%$ ( 12 hombres y 4 mujeres). Durante el segundo gobierno tripartito (2006 - 2010), presidido por el también socialista José Montilla, la presencia femenina se incrementa hasta el 28.5\% (10 consellers y 4 conselleres). Las elecciones de noviembre de 2010, crean un nuevo gobierno presidido por el convergente Artur Mas (Convergència $i$ Unió, $\mathrm{CiU}$ ), en el que la presencia femenina disminuye ligeramente respecto a la legislatura anterior, situándose en el 27.2\% ( 8 hombres y 3 mujeres).

El presente trabajo de investigación se propone analizar el tratamiento en prensa escrita de los consellers y conselleres que conforman el actual gobierno de la Generalitat. Este análisis nos ayudará a determinar si hay diferencias notables en el tratamiento que la prensa da a la cobertura de las mujeres políticas, en qué consisten estas diferencias y si existen diferencias entre los medios analizados en función de su adscripción ideológica.

\section{Metodología}

Este estudio examina la cobertura de los consellers y conselleres del gobierno de la Generalitat formado en diciembre de 2010, y que cuenta con tres mujeres: Joana Ortega (Vicepresidenta y titular del departamento de Gobernación y Relaciones Institucionales), Irene Rigau (Educación) y Pilar Fernández (Justicia). La muestra de estudio son los cinco diarios españoles con mayor circulación (EGM, 2011) y que además cubren el espectro ideológico izquierda - derecha: El Periódico de Catalunya (izquierda), El País (centro-izquierda), La Vanguardia (centro-derecha), El Mundo (derecha) y $A B C$ (conservador) (ver Reig, 1998: 95). Son estos diarios, con líneas editoriales diferenciadas, por tanto con públicos objetivos diferentes, los que tienen el mayor potencial para impactar sobre la audiencia y actuar como líderes de opinión en términos de establecimiento de agenda en el resto de medios (ver Kahn y Goldenberg, 1991; López García, 2004; Shoemaker y Reese,1996).

El periodo analizado abarca desde el 22 de diciembre de 2010 hasta el 5 de enero de 2011. Este periodo comprende la semana anterior y posterior al nombramiento oficial de los miembros del gobierno que formarán la IX legislatura. Se analiza este periodo ya que la cobertura realizada por la prensa de los primeros días de la formación de un gobierno ayuda a encuadrar la cobertura posterior de los miembros del Govern, al presentar los medios a los lectores "las primeras impresiones del carácter, experiencia, y formación de los políticos" (Norris, 1997: 157). 
La unidad de análisis es el artículo, que engloba tanto artículos de información, como editoriales y artículos de opinión. Los artículos han sido seleccionados a través de la base de datos Factiva, utilizando el nombre de los 11 miembros del Govern. Una vez los artículos son recopilados se eliminan los artículos duplicados y aquellos que mencionan al conseller o consellera de pasada sin tener ninguna relación con el contenido global del artículo. Estos artículos no son utilizados para el análisis final. El número total de piezas incluido en la muestra es de 500 artículos $^{2}$.

Las categorías utilizadas para codificar las diferencias entre hombres y mujeres políticos fueron tomadas de investigaciones anteriores (Aday y Devitt, 2001; Falk, 2008; Heldman et al., 2005; Kahn, 1996; Kittilson y Fridkin, 2008; Miller et al., 2009; Scharrer, 2002; Trimble, 2007). Las categorías desarrolladas incluyen variables demográficas (nombre del político, género, publicación, fecha publicación, sección, página) así como un conjunto de categorías diseñadas para identificar la representación de cada miembro del Govern. Estas categorías incluyen visibilidad (cantidad de cobertura), menciones al estado civil, edad, familia, apariencia y experiencia profesional; menciones al género y la asociación con una figura masculina; número de declaraciones representadas en las noticias; características de la personalidad (positivas o negativas), y el tono general de la cobertura (positiva, neutral, mixta, negativa). Para determinar la existencia de tendencias en la cobertura de los medios se utiliza el programa estadístico SPSS para comparar los datos.

Los primeros estudios que analizan la representación de la mujer política en los medios se centran en cuantificar la cantidad de cobertura que ésta recibe, analizando de esta manera su visibilidad o invisibilidad. Los resultados de los primeros estudios muestran que los medios ofrecen una menor cobertura de las mujeres que de los hombres que optan a un cargo político, incluso aunque éstas estén mejor posicionadas en los sondeos de opinión (ver Aday y Devitt, 2001; Heldman et al., 2005; Kahn, 1992, 1994, 1996; Kahn y Goldenberg, 1991; Serini et al.,1998). Por ejemplo, un estudio de Kahn (1996) en el que se analiza la cobertura de diferentes elecciones al Senado estadounidense durante los años 80 , señala que los hombres obtienen una media de 13 párrafos publicados al día, mientras que las mujeres obtienen sólo 10, una diferencia que, aunque no es muy pronunciada, se mantiene constante en todas las elecciones analizadas por la autora. Esta diferencia no sólo se da entre las candidatas sino también entre las mujeres que acceden a un cargo (ver Norris, 1997).

No obstante, estudios más recientes, sugieren que la cantidad de cobertura de hombres y mujeres se ha equilibrado y las diferencias podrían haber desaparecido (Kittilson y Fridkin, 2008; Semetko y Boomgaarden, 2007). Incluso la novedad que supone la participación de una mujer en la escena política podría provocar que ésta ganase mayor visibilidad que un hombre en las mismas circunstancias (Banwart et al., 2003; Bystrom et al., 2001; Jalalzai, 2006; Trimble, 2007). Así, en un análisis de las elecciones al Senado estadounidense durante los años 1992 y 2000 Jalalzai (2006) halla que la prensa escrita dedica más párrafos a las candidatas que a los candidatos: las

2101 artículos corresponden a El País, 46 a El Mundo, 95 a ABC, 134 a La Vanguardia, y 124 a El Periódico de Catalunya. Estos dos últimos periódicos presentan un mayor volumen de artículos debido a que son diarios editados en Cataluña. 
mujeres reciben una media de 70 párrafos a la semana, mientras que los hombres reciben una media de 41 párrafos.

En el presente estudio, la visibilidad es medida de dos formas diferentes. Por un lado, se cuantifican los artículos (informativos y de opinión) en los que los políticos son mencionados y, a continuación, se contabilizan los titulares en los que son citados (Aday y Devitt, 2001; Heldman et al., 2005; Kittilson y Fridkin, 2008; Miller et al., 2009; Trimble, 2007).

Otra variable a tener en cuenta es la presencia de menciones a la imagen, físico, edad y vida personal de los políticos (estado civil y familia). Diversos estudios apuntan que la prensa dedica más espacio a describir la apariencia de las mujeres políticas, cuestión que no sucede en la cobertura de los hombres políticos, centrándose la prensa principalmente en el ámbito profesional (Fernández, 2008; Ross, 2000). Un ejemplo reciente lo encontramos en la publicación de unas fotografías de la ministra de Sanidad española, Leire Pajín, en bikini, en las que se cuestiona su figura, o en las referencias constantes al físico de la política estadounidense Sarah Palin. Incluso algunos autores sugieren que la gran atención mostrada por parte de los medios al físico de las mujeres podría haber obligado a éstas a "adoptar una determinada imagen corporativa para no desentonar en el mundo de la política" (Van Acker, 2003: 131). Cuando las mujeres no acatan esta norma pueden ver como los medios dedicarán una gran cantidad de información a su forma de vestir y apariencia. El revuelo mediático que se formó por el traje utilizado por la ministra de Defensa española, Carme Chacón durante la Pascua Militar de 2009 sería un buen ejemplo de ello, así como las páginas que llenó el vestido lucido por la cancillera alemana, Angela Merkel, en la gala de apertura de la Ópera de Oslo en $2008^{3}$.

La edad también es destacada en la representación de las mujeres políticas. Mientras la edad para los hombres políticos comporta distinción y realización profesional, una mujer entrada en años ha de hacer frente a una discriminación cultural que trata de invisibilizar (e incluso rechazar) a las mujeres de cierta edad (Lawrence y Rose, 2010). Un ejemplo fueron las constantes referencias peyorativas a la edad de Hillary Clinton durante las primarias del Partido Demócrata estadounidense (ver Traister, 2007).

Los medios también tienden a identificar a las mujeres políticas en términos de su estado civil o familiar, identificación que no se encuentra cuando los medios cubren a políticos varones (Banwart et al., 2003; Braden, 1996; Bystrom et al., 2001; Falk, 2008; Jamieson, 1995). Así, un reciente análisis realizado por Falk (2008) sobre la cobertura en prensa de ocho destacadas candidatas a la presidencia norteamericana desde 1872 hasta 2004, encuentra que es más probable que las candidatas obtengan mayor número de menciones a su familia por parte de la prensa que los candidatos varones.

Para analizar las referencias a la vida personal, por un lado, codifico como una referencia a la apariencia el número de artículos que realizan cualquier mención al físico o a la forma de vestir de los y las políticas. También son cuantificados los artículos en los que se hace referencia al estado civil o al cónyuge del miembro del Govern, así

3 ‘¿Chacón como Andy Warhol? (La Vanguardia, 9 Enero 2009), 'Elogio del poder blando' (ABC, 15 Abril 2008), 'Ministra de Sanidad, mujer sana' (El Mundo, 9 Julio 2011). 
como los que hacen referencia a su edad o generación, y a sus lazos familiares (hijos y padres) (ver Falk, 2008; Miller et al., 2009; Trimble, 2007).

Otro aspecto es la representación de la experiencia profesional de las mujeres políticas. Por ejemplo, Falk (2008) señala que las mujeres tienen una mayor probabilidad de ver desaparecidos sus cargos profesionales en los artículos sobre ellas, mientras que los hombres sí son vinculados a su cargo. Siguiendo con la candidatura de Hillary Clinton, los medios subestimaron su experiencia profesional, omitiendo repetidamente su cargo de Senadora, y siendo destacada por los medios su función de primera dama (función asociada principalmente a actividades protocolarias que ocupa mientras su marido se encuentra al frente de la presidencia estadounidense) (ver Falk, 2008; Fernández, 2010; Stein, 2009). Este dato, unido al hecho que las mujeres obtengan más menciones a su vida familiar, provoca la asociación de las mujeres "con el hogar y de los hombres con el trabajo, afectando la forma en que las candidatas son encuadradas, reforzando este tipo de encuadre los estereotipos de género" (Falk, 2008: 62). En este estudio, se codifican todos los artículos en los que se menciona la experiencia profesional o se hace referencia a anteriores cargos ostentados.

Norris (1997) halla que el género de las mujeres políticas aparece como primer descriptor cuando los medios hablan de ellas. En cambio, los hombres tienen más posibilidades de ser descritos en términos neutrales de género, es decir, con el género como irrelevante (ver también Fernández, 2010). Por ejemplo, un análisis de las elecciones presidenciales chilenas de 2005, ganadas por la candidata de la Concertación de Partidos por la Democracia, Michelle Bachelet, destaca que, mientras el 13\% de los artículos aparecidos en la prensa escrita mencionan el género de Bachelet, ninguno alude al género de los otros candidatos (todos varones) (Valenzuela y Correa, 2006). Para analizar si el género juega un papel importante en la representación de las mujeres políticas, se codifican los artículos en los que se menciona explícitamente el género.

En muchas ocasiones también se relaciona a las mujeres con una figura masculina, que va más allá del cónyuge, asociación que iría en detrimento de la aceptación como propios de los logros de las mujeres políticas (Trimble, 2006; Van Zoonen, 2006). Baider (2007) en su estudio sobre la representación en prensa de diferentes mujeres con cargo político, halla que todas son definidas por su relación con algún varón: la ex-presidenta chilena, Michelle Bachelet, como hija de un militar asesinado por la dictadura; Ellen Johnson-Sirleaf, presidenta de Liberia, como hija de un legislador nacional; Ségolène Royal, candidata del Partido Socialista Francés a las elecciones presidenciales de 2007, como mujer de político e hija de un militar; e incluso Angela Merkel, actual cancillera alemana, es definida como 'hija espiritual' de Helmut Kohl. Para averiguar si la figura masculina de los integrantes del Govern es citada en relación al género de éstos, se cuantifican los artículos en los que se hace referencia a un mentor o figura prominente, anotando el género de éste.

También se analiza la forma en que las mujeres son nombradas en los medios. Si los medios emplean una cobertura sin distinción de género, deberían nombrar a los políticos de la misma forma, sin diferencias en función de su género. Ya que los nombres que los periodistas utilizan para referirse a los políticos dibujan una sutil foto de éstos, el nombre utilizado por los medios constituirá un interesante indicador del estatus dado a los y las políticas (Uscinski y Goren, 2011). Existen estudios que apun- 
tan una probabilidad mayor de que los medios se refieran a las mujeres en términos familiares por su nombre de pila o con un diminutivo, lo que puede tener el efecto de rebajar el estatus de las mujeres e influir en la conducta de los lectores (Falk, 2008; Fernández, 2008, 2010; Rittau, 2008; Sreberny-Mohammady y Ross, 1996). Por ejemplo, Baider (2008), al analizar la representación en la prensa francesa de Bachelet (Chile), Sirleaf (Liberia), Merkel (Alemania) y Royal (Francia), apunta una tendencia general a utilizar el nombre de pila para referirse a ellas. Es más, un análisis más detallado encuentra que, en el caso de Ségolène Royal, cuando se utiliza su apellido, en el 50\% de las ocasiones se utiliza como adjetivo ('la vague Royal', 'le peril Royal'), en el $30 \%$ de las ocasiones aparece unido al apellido de un político varón ('Fabius et Royal'), y sólo en el $20 \%$, el apellido es utilizado para referirse a la política francesa en solitario (Baider, 2008: 32).

Como es práctica habitual en el periodismo que se presente a las personas en primer lugar por su nombre completo y, posteriormente, utilizando otras menciones más reducidas, en el presente estudio se codifica exclusivamente la primera mención en los artículos de los miembros del Govern, utilizando las siguientes categorías: "nombre de pila", "apellido", "nombre + apellido", y "otros".

Por otro lado, las mujeres tienen una probabilidad menor de ver sus declaraciones citadas en los medios (Comas D'Argemir, 2009). Un estudio realizado por el Consell de l'Audiovisual de Catalunya (2009) señala que el tiempo de palabra en los informativos televisivos de las mujeres políticas es de un $15.5 \%$, un porcentaje inferior a su presencia real en la política. Además, la duración de las intervenciones de los hombres políticos ( 2 minutos y 1 segundo) prácticamente duplica la de las mujeres políticas (1 minuto y 12 segundos). Ya que las declaraciones realizadas a la prensa son una forma mediante la que los ciudadanos obtienen información directa sobre la clase política y, al mismo tiempo, los políticos tienen acceso directo a los ciudadanos (Falk, 2008), cuantificar las declaraciones representadas en la prensa servirá como indicador de las diferencias entre hombres y mujeres políticos en los medios.

A medida que aumenta el proceso de personalización de la política, los rasgos de personalidad asociados con la clase política son enfatizados en mayor medida por los medios a expensas de otras cuestiones (ver Aalberg y Jenssen, 2007). Por lo tanto, si los votantes obtienen una gran cantidad de información sobre los rasgos de personalidad de los candidatos y candidatas, tendrán en consideración esta información cuando desarrollen sus impresiones sobre éstos, pero si los periodistas tienden a describir los rasgos de personalidad de hombres y mujeres de forma diferente, esta diferencia tendrá consecuencias también sobre sus evaluaciones (Kahn, 1996: 53).

En los primeros estudios sobre la representación de las mujeres políticas se diferencia entre rasgos 'masculinos' y rasgos 'femeninos', categorización que tiene sus

${ }^{4}$ Los rasgos estereotipadamente 'masculinos' incluyen la experiencia, el liderazgo, la capacidad de trabajo, la vitalidad, la competitividad, el razonamiento, el conocimiento, la agresividad, la ambición o la independencia, entre otros. Los rasgos estereotipadamente 'femeninos' incluyen la honestidad, la compasión, la sensibilidad, la debilidad, la ingenuidad, la pasividad, la emotividad, la simpatía o la dependencia (ver Bem, 1974; Dolan, 2004; Kahn, 1996; Kittilson y Fridkin, 2008). 
raíces en los estereotipos de género. En líneas generales, estos estudios hallan que los periodistas tienden a discutir las categorías de la personalidad 'masculina' de forma más frecuente que las características 'femeninas' (Kahn, 1994), asumiendo que las características masculinas son las más apropiadas para asumir el poder político (Carroll y Fox, 2006). No obstante, y dado que no todos los rasgos 'femeninos' han de ser negativos ni todos los rasgos estereotipadamente 'masculinos' han de ser positivos para un cargo político, y que el contexto en el que tiene lugar las elecciones determina en gran medida los atributos deseables, recientes estudios realizan una dicotomía entre rasgos favorables, donde tendrían cabida rasgos 'femeninos', y rasgos 'negativos', donde también tendrían cabida rasgos 'masculinos' (Fernández, 2010; Heldman et al., 2005; Miller et al., 2009; Van Acker, 2003). Por ejemplo, Miller et al. (2009) analizan los rasgos de personalidad con los que son asociados Barack Obama y Hillary Clinton en el proceso de primarias del Partido Demócrata y hallan que los rasgos de personalidad de Clinton son más negativos que los de Obama, rasgos centrados básicamente en su personalidad (Clinton es mostrada como hermética, engañosa, polarizante, fría y calculadora) (ver también Fernández, 2010). En el presente estudio se codificarán los rasgos de personalidad siguiendo la metodología elaborada por MILLER et al. (2009), codificando entre características de personalidad 'positivas' y 'negativas' y clasificando los rasgos en función del contexto de cada artículo.

Finalmente, y estrechamente relacionado con los rasgos de personalidad, se analiza el tono de la cobertura empleada por los medios para representar a los miembros del Govern. Diferentes estudios sugieren que la prensa cuestiona en mayor medida la capacidad de las mujeres, haciendo servir para ello un tono de cobertura más negativo que cuando representa a hombres políticos (Fernández, 2008, 2010; Kahn, 1992, 1994; Kahn y Goldenberg, 1991). Siguiendo con Hillary Clinton, Miller et al. (2009) hallan que cuando Clinton está en lo más alto de los sondeos de opinión los titulares sobre ella muestran un tono menos favorable.

En el presente estudio se codifica el tono del artículo siguiendo la metodología empleada por Scharrer (2002, ver también Fernández, 2010; Kittilson y Fridkin, 2008). El artículo se codifica como positivo si la pieza contiene de forma clara palabras que retratan al político o política favorablemente; mixto, si el artículo contiene un tono ambivalente; neutral, si el artículo es equilibrado o no contiene críticas positivas ni negativas; y se codifica como negativo si el artículo contiene de forma clara palabras que expresan desaprobación hacia el político o política.

\section{Resultados}

\subsection{Visibilidad}

El Govern catalán surgido de las elecciones llevadas a cabo el 28 de noviembre de 2010 está formado por 11 miembros, 3 mujeres y 8 hombres, lo que supone un porcentaje de $72.7 \%$ hombres y $27.2 \%$ mujeres. Si observamos la Tabla 1 , al analizar la cantidad de cobertura recibida por hombres y mujeres, las conselleres obtienen un porcentaje menor a su presencia real en el Govern, lo que resultaría llamativo porque, por primera vez, una mujer, Joana Ortega, ocupa el cargo de vicepresidenta. Anteriores estudios habían destacado la novedad como uno de los factores que ayudan a las mujeres políticas a tener visibilidad en los medios, pero en este caso no parece que haya sido así. 
Joana Ortega obtiene el 9.6\% de las menciones en el total del Govern, siendo eclipsada por otros consellers varones. El conseller de Cultura, Ferran Mascarell (21.0\%) es el conseller que más cobertura recibe por parte de los medios, a pesar de ostentar una cartera considerada de menor prestigio. Es seguido del conseller de Interior, Felip Puig (15.8\%), y de Economía y Conocimiento, Andreu Mas-Colell (11.6\%). El interés que despierta la entrada de Mascarell, antiguo conseller de Cultura durante el primer gobierno del Tripartit, puede haber provocado esta gran atención por parte de los medios hacia su persona. Las otras dos conselleres, Irene Rigau (Educación) y Pilar Fernández (Justicia) obtienen una visibilidad del 5.8\% y del 8.2\%, respectivamente. Esta visibilidad se podría conectar con el prestigio de las conselleries, siendo aquellas con mayor prestigio (Interior o Economía y Conocimiento) las que obtienen una mayor cobertura, mientras que las conselleries con menor prestigio (Bienestar Social y Familia o Educación) obtienen una menor cobertura.

Tabla I. Visibilidad y género (\%). Porcentajes en las columnas con el número de menciones en paréntesis

\begin{tabular}{|l|c|c|c|}
\hline Diarios & Consellers & Conselleres & Diferencia \\
\hline El País & $78.2(79)$ & $21.8(22)$ & 56.4 \\
\hline El Mundo & $73.9(34)$ & $26.1(12)$ & 47.8 \\
\hline La Vanguardia & $76.9(103)$ & $23.1(31)$ & 53.8 \\
\hline ABC & $78.9(75)$ & $21.1(20)$ & 57.8 \\
\hline El Periódico & $73.4(91)$ & $26.6(33)$ & 47.3 \\
\hline Total & $76.4(382)$ & $23.6(118)$ & 52.8 \\
\hline Composición Govern & $72.7(8)$ & $27.2(3)$ & \\
\hline
\end{tabular}

En cuanto a la presencia de los miembros del Govern en los titulares de los medios analizados, el $19.4 \%$ de los artículos que menciona a los consellers varones $(\mathrm{n}=382)$ lleva el nombre de uno de ellos en el titular, mientras que el nombre de una consellera aparece sólo en el $8.5 \%$ de los artículos en los que son mencionadas $(\mathrm{n}=118)$.

$\mathrm{Al}$ analizar por medio, El Periódico de Catalunya (26.6\%) y El Mundo (26.1\%) son los diarios que más espacio dedican a las conselleres, mientras que $A B C(21.1 \%)$ y $E l$ País (21.8\%) son los que menos. La Vanguardia es el diario que más espacio dedica a Joana Ortega (11.9\% de la cobertura), pero todos los medios centran principalmente la atención en el nombramiento de Ferran Mascarell como conseller de Cultura.

\subsection{Vida personal y profesional}

$\mathrm{Al}$ analizar la cobertura personal que reciben los consellers y conselleres no se hallan diferencias significativas entre hombres y mujeres. Ambos reciben pocas menciones a su estado civil, apariencia y familia, aunque las conselleres reciben casi el doble de referencias a su familia que los consellers ( $2.5 \%$ y $1.3 \%$, respectivamente). Las referencias a la edad son numerosas, con un porcentaje ligeramente superior en el caso de las conselleres ( $8.5 \%$ vs. $8.1 \%$ ), pero no existen diferencias significativas entre ambos géneros. 
También se analiza las referencias a la experiencia profesional. Los consellers obtienen un porcentaje mayor de artículos en los que se cita su experiencia $(30.1 \%$ vs. $26.3 \%$ ), pero no es ésta tampoco una diferencia estadísticamente significativa.

Entre los medios analizados, se ha de destacar el diario conservador El Mundo, que cita la experiencia profesional en 58.8\% de los artículos sobre los consellers varones, mientras sólo lo hace en el $25 \%$ de los artículos sobre las conselleres. En el resto de medios, las diferencias no son tan acusadas, e incluso El País y $A B C$ citan en mayor medida la experiencia de las conselleres por encima de la de los consellers (31.8\% vs. $27.8 \%$ en el caso de El País, y $20.0 \%$ vs. $18.7 \%$ de $A B C$ ).

\subsection{Género y figura masculina}

Al contabilizar los artículos en los que se menciona explícitamente el género, el análisis muestra una diferencia significativa entre hombres y mujeres. El $11.9 \%$ de los artículos que menciona a conselleres, destaca su género, mientras que sólo lo hace el $0.3 \%$ (un único artículo) en los que se menciona a los consellers.

Por medio analizado, El Mundo es el diario que más destaca el género de las conselleres en su cobertura (25.0\%), mientras que El Periódico es el diario que menos referencias realiza $(6.1 \%)$.

Tabla II. Menciones al género y asociación con una figura masculina (\%). Las entradas representan el porcentaje de menciones asociado a un género específico del gabinete, basado

en el número total de artículos mencionando a cada género. ${ }^{*} \mathrm{p}<.1 ;{ }^{*} \mathrm{p}<.05 ;{ }^{*} *{ }^{*} \mathrm{p}<.01$

\begin{tabular}{|l|c|c|c|}
\hline & Género & Figura Masculina & (N) \\
\hline Hombres & $3 \%$ & $2.1 \%$ & $(382)$ \\
\hline Mujeres & $11.9 \%$ & $8.5 \%$ & $(118)$ \\
\hline Diferencia & $-11.6^{* * *}$ & $-6.4 \% * * *$ & \\
\hline
\end{tabular}

Respecto a la asociación de las mujeres políticas con una figura masculina, el 8.5\% de los artículos que nombran a una consellera se hace mención a un mentor. En el caso de los consellers, sólo es destacada la figura de mentor en $2.1 \%$ de los artículos en los que son citados. Curiosamente, el porcentaje de artículos positivos cuando se destaca una figura masculina como mentor alcanza el $60 \%$ de los artículos que citan a las conselleres, con un $20 \%$ de artículos neutrales y un $20 \%$ con un tono negativo. En el caso de los consellers, la mitad de los artículos obtienen un tono neutral, un $37.5 \%$ positivo, y sólo un $12.5 \%$ un tono negativo.

Por medio, todos los diarios relacionan en mayor medida a las conselleres con una figura masculina, siendo El País (13.6\%), La Vanguardia (9.7\%) y El Mundo (8.3\%) los medios donde la asociación se realiza en un porcentaje mayor. El Mundo es también el diario que asocia en mayor medida a los consellers con una figura masculina (5.9\%).

\subsection{Primer nombre}

$\mathrm{Al}$ analizar el primer nombre que utilizan los medios para referirse a los miembros del Govern encontramos una diferencia estadísticamente significativa entre hombres y mujeres en la forma en que éstos son nombrados. No hallamos ningún artículo en la 
muestra analizada en el que se refiera a los miembros del Govern por su nombre de pila. Los medios se refieren a ellos utilizando su apellido (13.9\%) o su nombre y ape1lido (86.2\%). No obstante, como muestra la Tabla 3, aunque la prensa utiliza principalmente la fórmula "nombre + apellido", existe una diferencia estadísticamente significativa de 8.1 puntos porcentuales entre hombres y mujeres.

Las entradas representan el porcentaje de menciones asociado a un género específico del gabinete, basado en el número total de artículos mencionando a cada género.

Tabla III. Primer nombre utilizado por los medios $(\%)^{*} \mathrm{p}<.1 ; * * \mathrm{p}<.05 ; * * * \mathrm{p}<.01$

\begin{tabular}{|l|c|c|c|}
\hline & Apellido & Nombre + Apellido & (N) \\
\hline Hombres & 15.7 & 84.3 & 382 \\
\hline Mujeres & 7.6 & 92.4 & 118 \\
\hline Diferencia & $8.1^{* *}$ & $-8.1^{* *}$ & \\
\hline
\end{tabular}

Al ser práctica habitual en el periodismo que se presente en los artículos a las personas en primer lugar por su nombre completo para posteriormente utilizar otras nominaciones, resulta interesante observar que los medios utilizan únicamente el apellido de los consellers en una porcentaje significativamente superior al uso del apellido de las conselleres ( $15.7 \%$ vs. $7.6 \%)$. Este dato se podría explicar por la familiaridad de los medios con los consellers varones, mientras que las conselleres, más desconocidas por los medios, son tratadas con mayor formalidad cuando son nombradas, empleando para ello su nombre completo. O quizás también existe la necesidad por parte de los periodistas de utilizar el nombre de las conselleres con el fin de destacar que quien ocupa el cargo es una mujer.

\subsection{Declaraciones}

Como se observa en la Tabla 4, los consellers tienen más opciones de ver su discurso representado en las noticias. El discurso de los miembros varones del Govern es citado en $11.8 \%$ de los artículos en los que son nombrados, mientras que el discurso de las conselleres es citado únicamente en $4.2 \%$ de los artículos.

Tabla IV. Declaraciones de los conselleres y conselleres (\%) Porcentaje en columnas con base $\mathrm{n}$ en paréntesis. ${ }^{*} \mathrm{p}<.1^{* *} \mathrm{p}<.05 ; * * * \mathrm{p}<.01$

\begin{tabular}{|l|c|c|c|}
\hline Diarios & Hombres & Mujeres & Diferencia \\
\hline El País & $22.8(18)$ & $4.5(1)$ & $18.3^{*}$ \\
\hline El Mundo & $-(0)$ & $-(0)$ & 0 \\
\hline ABC & $16.0(12)$ & $10.0(2)$ & 6.0 \\
\hline La Vanguardia & $6.8(7)$ & $3.2(1)$ & 3.6 \\
\hline El Periódico & $8.8(8)$ & $3.0(1)$ & 5.8 \\
\hline Total & $11.8(382)$ & $4.2(118)$ & $7.6^{* *}$ \\
\hline
\end{tabular}

Los datos muestran que todos los periódicos dedican mayor espacio a representar las declaraciones de los hombres políticos (menos El Mundo, que no reproduce ninguna cita de los miembros del govern en sus artículos). La mayor diferencia se encuentra en el diario de centro-izquierda El País, donde las declaraciones de los 
consellers son representadas en más de una quinta parte de los artículos en los que son citados, mientras que las declaraciones de las conselleres sólo se hallan en el $4.5 \%$ de sus artículos.

\subsection{Características personales}

En la cobertura que los medios analizados dedican a las conselleres, éstas son representadas, en mayor medida incluso que los consellers, con rasgos de personalidad positivos (e.g., trabajadora, con experiencia, competente, rigurosa). Pero también es cierto que, al mismo tiempo, reciben un mayor porcentaje de rasgos negativos que sus colegas varones (especialmente dirigidos al fulgurante ascenso o a la carrera meteórica de algunas conselleres), aunque esta diferencia no es estadísticamente significativa.

Por medios, El Mundo no dedica ningún rasgo positivo a las conselleres (un 2.9\% a los miembros varones). El diario que más porcentaje de rasgos positivos dedica a hombres y mujeres es El País (12.7\% y $18.2 \%$, respectivamente). En cuanto a los rasgos negativos, ni $A B C$ ni El Periódico hacen referencia alguna a rasgos negativos en su cobertura. El Mundo es el que mayor porcentaje dedica a los rasgos negativos de las conselleres y de los consellers (8.3\% y 5.9\%, respectivamente).

\subsection{Tono de la cobertura}

El análisis realizado muestra que las conselleres reciben un tono de cobertura principalmente neutral (46.6\%), seguido de un tono positivo $(22.0 \%)$ y un tono negativo (20.3\%). Como muestra la Tabla 5, resultan estadísticamente significativas las diferencias con los consellers en el tono positivo (30.4\% vs. $22.0 \%)$, en el tono mixto ( $7.1 \%$ vs. $3.4 \%$ ) y, especialmente, en el tono negativo: mientras que sólo $8.4 \%$ de los artículos sobre los consellers tiene un tono negativo, en el caso de las conselleres alcanza el $20.3 \%$ de los artículos en los que éstas son citadas.

Tabla V. Tono de la cobertura (\%). Las entradas representan el porcentaje de menciones asociado a un género específico del gabinete, basado en el número total de artículos mencionando a cada género.

El valor $\mathrm{p}$ se basa en la diferencia en el test de porcentaje. ${ }^{*} \mathrm{p}<.1 ;{ }^{*} \mathrm{p}<.05 ;{ }^{*} * \mathrm{p}<.01$

\begin{tabular}{|l|c|c|c|c|c|}
\hline & Positivo & Neutral & Mixto & Negativo & $(\mathbf{N})$ \\
\hline Hombres & 30.4 & 44.8 & 7.1 & 8.4 & $(382)$ \\
\hline Mujeres & 22.0 & 46.6 & 3.4 & 20.3 & $(118)$ \\
\hline Diferencia & $8.4^{*}$ & -1.8 & $-3.7^{*}$ & $-11.9^{* * *}$ & \\
\hline
\end{tabular}

$\mathrm{Al}$ analizar por diario, todos emplean principalmente un tono neutral en sus artículos, excepto La Vanguardia que utiliza un tono positivo con los miembros varones (49.5\%). El medio que dedica un tono más negativo a las conselleres es El Periódico de Catalunya (33.3\% de la cobertura tiene un tono negativo), seguido de El Mundo (25.0\%) y El País (22.7\%). El Mundo es también el diario que dedica un tono más negativo a los consellers (20.6\%). 


\section{Conclusiones}

Este estudio preliminar sugiere que la aún desigual presencia de las mujeres en la vida política catalana va pareja a una también desigual representación de éstas en la prensa escrita. El género de las mujeres políticas continúa siendo considerado relevante en los medios. Y las mujeres tienen además una mayor probabilidad de ser asociadas a una figura masculina que les ha servido de mentor, lo que envía el sutil mensaje de que no están en la carrera política por sus propios méritos sino gracias a la ayuda de alguien, un mentor masculino.

Otro importante hallazgo del estudio es la diferencia entre hombres y mujeres en la cantidad de declaraciones representadas en la prensa. Los resultados muestran que las mujeres políticas presentan una menor probabilidad de ver su discurso representado en los medios, lo que puede enviar el mensaje de que no tienen nada que decir. No obstante, al igual que con la visibilidad de las conselleres, se hace necesario un análisis más profundo para averiguar si el prestigio de la cartera que se ocupa estaría también relacionado con una mayor o menor representación de sus titulares en los medios.

La diferencia en la forma de nombrar a los miembros del Govern en función de su género también es un indicador de los diferentes registros utilizados cuando una mujer entra en el ámbito político. ¿Se trata de un desconocimiento de los medios de estas mujeres o de querer destacar que éstas son mujeres mediante el uso de su nombre?

Finalmente, aunque predomina un tono neutral (46.6\%) en los artículos donde son citadas las conselleres, éstas reciben un tono más negativo que sus colegas varones, lo que confirmaría anteriores estudios que sugieren que las mujeres políticas reciben un tono de cobertura más negativo.

No obstante, algunos indicadores apuntan a una mejora en la cobertura de los medios. Así, aunque son asociadas a rasgos negativos en mayor medida que sus colegas varones, el análisis también revela que las conselleres también son asociadas a rasgos positivos en mayor proporción. Por otro lado, no se han hallado diferencias estadísticamente significativas en las menciones al estado civil, apariencia, edad, o familia. Y la diferencia en la cantidad de menciones a la experiencia profesional entre hombres y mujeres tampoco resulta estadísticamente significativa.

En cuanto a las diferencias entre los medios que conforman la muestra, los datos hallados sugieren que la representación de las mujeres políticas no se encuentra influenciada por la ideología del medio (progresista - conservador). Más bien, convendría estudiar en mayor grado cómo las rutinas y lógicas periodísticas enraizadas en las redacciones de todos los medios hacen que éstos utilicen los estereotipos de género de forma no consciente en su trabajo (ver Shoemaker y Reese, 1996).

Este estudio ha sido una primera aproximación a la forma en que la prensa escrita ha representado la formación de un gobierno autonómico. Se hace necesario ampliar los resultados obtenidos en este estudio con análisis de la representación de otros gobiernos en sus primeros días de andadura para poder localizar posibles patrones que se repitan en diferentes contextos políticos. Por otro lado, el presente estudio se ha centrado exclusivamente en el análisis de las noticias aparecidas en prensa escrita. Existe una falta de estudios que aborden la representación de las mujeres en otros medios (especialmente, la blogosfera), donde noticia y opinión se confunden y puede ser más 
fácil encontrar observaciones gratuitas sobre la apariencia personal y referencias manifiestamente sexistas (Lawrence y Rose, 2010).

Además, se hace necesario analizar las consecuencias de este tipo de cobertura sobre la ciudadanía. ¿Qué efecto tiene una cobertura donde el género de las mujeres políticas es importante?¿Qué efecto tiene esta cobertura no sólo entre la ciudadanía sino también en la decisión de otras mujeres a presentarse como candidatas a un cargo político? Todas estas cuestiones invitan a continuar estudiando la forma en que los medios representan a las mujeres que forman, o quieren formar parte, de los espacios de decisión política.

\section{Referencias Bibliográficas}

AALBERG, Toril y JENSSEN, Anders Todal (2007): "Gender stereotyping of political candidates: An experimental study of political communication", en Nordicom Review, vol. 28, n 1, pp. 17-32.

ADAY, Sean y DEVITT, James (2001): "Style over Substance: Newspaper coverage of Elizabeth Dole's Presidential bid", en Harvard International Journal of Press / Politics, vol. 6, n 2, pp. 52-73.

BAIDER, Fabienne (2007): "Feminisation in Linguistics, Women in Politics: How is the French Press Coping?", en International Perspectives on Gender and Language: Cuaderno da langage e sexo, $\mathrm{n}^{\circ} 3$, pp. 770-794.

BAIDER, Fabienne (2008). "Substantive disembodiment, syntactic embedment or the null effect of 'feminist' lexical change", En HOLMES, J. y MARRA, M. (eds.): Proceedings of the $5^{\text {th }}$ Biennial International Gender and Language Asociation Conference. Wellington, New Zealand, Victoria University of Wellington, pp.149-156.

BANWART, Mary C.; BYSTROM Dianne G.; y ROBERTSON, Terry (2003): "From the primary to the general election: a comparative analysis of candidate media coverage in mixed-gender 2000 races for governor and US Senate", en American Behavioral Scientist, vol. 46, nº5, pp. 658-676.

BRADEN, Maria (1996): Women politicians and the media. Lexington, University Press of Kentucky.

BYSTROM, Dianne; ROBERTSON, Terry; y BANWART, Mary C. (2001): "Framing the fight: An analysis of media coverage of female and male candidates in primary races for Governor and US Senate in 2000", en American Behavioral Scientist, $\mathrm{n}^{\circ}$ 44, pp. 1999-2013.

CARROLL, Susan J., y FOX, Richard L. (2006): Gender and Elections: Shapping the Future of American Politics. Cambridge, Cambridge University Press.

COMAS D'ARGEMIR, Dolors (2009): "Les dones en els informatius de televisió", en Quaderns del CAC, n 33 , pp. 97-108.

CONSELL DE L'AUDIOVISUAL DE CATALUNYA (2009): Informe sectorial trimestral. La presència de les dones en la informació. Barcelona, Consell de l'Audiovisual de Catalunya. 
EGM (ESTUDIO GENERAL DE MEDIOS, 2011): "Resumen General de Resultados EGM. Febrero a Novembre de 2011", en Asociación para la Investigación de Medios de Comunicación: http://www.aimc.es/-Datos-EGM-Resumen-General-.html [fecha de consulta: 1 de abril de 2012]

FALK, Erika (2008): Women for President: Media bias in eight campaigns. Champaign, University of Illinois Press.

FERNÁNDEZ GARCÍA, Núria (2008): “La construcción mediática del líder político desde una perspectiva de género: análisis del tratamiento mediático de Ségolène Royal en las columnas de opinión de Le Figaro y Libération durante las elecciones primarias del partido socialista francés", en PÉREZ-AMAT, Ricardo; NÚÑEZ, Sonia; y GARCÍA JIMÉNEZ, Antonio (coord.): Comunicación, identidad y género. Madrid, Editorial Fragua, pp.481-493.

FERNÁNDEZ GARCÍA, Núria (2010): "Framing Hillary Clinton en la prensa española: ¿Candidata o Mujer?”, en Observatorio (OBS*) Journal, vol. 4, n 3, pp. 209228.

HELDMAN, Caroline; CARROLL, Susan J.;y OLSON, Stephanie (2005): "She brought only a skirt: Print media coverage of Elizabeth Dole's bid for the Republican presidential nomination", en Political Communication, vol. 22, nº 3, pp. 315335.

INE (INSTITUTO NACIONAL DE ESTADÍSTICA, 2011). Mujeres y Hombres en España. Madrid, Instituto Nacional de Estadística.

INTER-PARLIAMENTARY UNION (2011). "World Classification", en Women in national Parliaments: http://www.ipu.org/wmn-e/classif.htm [fecha de consulta: 1 de abril de 2012]

JALALZAI, Farida (2006): "Women candidates and the media: 1992 - 2000 elections", en Politics \& Policy, vol. 34, n³, pp. 606-633.

JAMIESON, Kathleen H. (1995): Beyond the double bind: Women and leadership. New York, Oxford University Press.

KAHN, Kim Fridkin (1992): "Does being a male help? An investigation of the effects of candidate gender and campaign coverage on evaluations of US Senate candidates", en Journal of Politics, vol. 54, n² 2, pp. 497-517.

KAHN, Kim Fridkin (1994): "The distorted mirror: Press coverage of women candidates for statewide office", en Journal of Politics, vol. 56, n 1, pp. 154-173.

KAHN, Kim Fridkin (1996): The political consequences of being a woman: How stereotypes influence the conduct and consequences of political campaigns. New York, Columbia University Press.

KAHN, Kim Fridkin y GOLDENBERG, Edie N. (1991): "Women candidates in the news: An examination of gender differences in US Senate campaign coverage", en The Public Opinion Quarterly, vol. 55, n 2, pp. 180-199. 
KITTILSON, Miki Caul y FRIDKIN, Kim (2008): "Gender, Candidate Portrayals and Election Campaigns: A Comparative Perspective", en Politics \& Gender, vol. 4, n ${ }^{\circ}$ 3, pp. 371-392.

LAWLESS, Jennifer L. (2009): "Sexism and gender bias in election 2008: A more complex path for women in politics", en Politics \& Gener, vol. 5, nº 1, pp. 70-80.

LAWRENCE, Regina G., y ROSE, Melody (2010): Hillary Clinton's Race for the White House. Gender Politics and the Media on the Campaign Trail, Boulder, Lynne Rienner Publishers.

LÓPEZ GARCÍA, Guillermo (2004): Comunicación electoral y formación de la opinion pública: las elecciones generales de 2000 en la prensa española. Valencia, Servei de Publicacions de la Universitat de Valencia.

MILLER, Melissa; PEAKE, Jeffrey; y BOULTON, Brittany (2009): "You've Come a Long Way Baby? Press Coverage of Hillary Clinton's Presidential Campaign", paper presentado en el Annual Meeting of the Midwest Political Science. Chicago, IL.

NORRIS, Pippa (1997): "Women leaders worldwide: A splash of color in the photo op", en NORRIS, Pippa (ed.): Women, Media, and Politics. New York, NY, Oxford University Press, pp. 149-165.

RITTAU, Andreas (2008): "Les cent jours d'Angela Merkel à travers deux articles du Monde", en Langage et Societé, vol. 2, n 124, pp. 113-126.

ROSS, Karen (2000): Framed: Women, politics and news media in a general election climate. Coventry, Centre for Communication, Culture and Media.

SCAMMELL, Margaret y SEMETKO, Holli A. (eds., 2000): The Media, Journalism and Democracy. Aldershot, Dartmouth.

SCHARRER, Erica (2002): “An 'Improbable Leap': a content analysis of newspaper coverage of Hillary Clinton's transition from first lady to Senate candidate", en Journalism Studies, vol. 3, n³, pp. 393-406.

SEMETKO, Holli A. y BOOMGAARDEN, Hajo G. (2007): "Reporting Germany's 2005 Bundestag election campaign: Was gender an issue?", en The International Journal of Press / Politics, ${ }^{\circ}$ 12, pp. 154-171.

SERINI, Shirley; POWERS, Angela; y JOHNSON, Susan (1998): "Of Horse Race and Policy Issues: A Study of Gender in Coverageof a Gubernatorial Election by Two Major Metropolitan Newspapers", en Journalism and Mass Communication Quarterly, vol. 75, nº 1, pp. 194-204.

SHOEMAKER, Pamela J., y REESE, Stephen D. (1996): Mediating the message: Theories of influences on mass media content. White Plains, NY, Longman.

SREBERNY, Annabelle, y VAN ZOONEN, Liesbet (eds., 2000): Gender, politics and communication. Cresskill, NJ, Hampton Press. 
SREBERNY-MOHAMMADI, Annabelle \& ROSS, Karen (1996): "Women MPs and the media: Representing the body politic", en Parliamentary Affairs, vol. 49, $\mathrm{n}^{\circ}$, pp. 103-115.

STEIN, Karen F. (2009): "The Cleavage Commotion: How the Press Covered Senator Clinton's Campaign", en SHECKELS, Theodore (ed.): Cracked but not shattered. Hillary Rodham Clinton's unsuccessful campaign for the presidency. Lanham, Maryland, Lexington Books, pp. 173-188.

TRAISTER, Rebecca (2007): "Campaign While Female", en Salon.com: http://www.salon.com/2007/12/19/wrinkled_hillary/[fecha de consulta: 1 de abril de 2012]

TRIMBLE, Linda (2007): "Gender, political leadership and media visibility: Globe and Mail coverage of Conservative party of Canada leadership contests", en Canadian Journal of Political Science, vol. 40, nº 4, pp. 969-993.

USCINSKI, Joseph E. y GOREN, Lilly J. (2011): "What's in a Name? Coverage of Senator Hillary Clinton during the 2008 Democratic Primary", en Political Research Quarterly, vol. 64, n 4, pp. 884-896.

VALENZUELA, Sebastián y CORREA, Teresa (2006): "Prensa y candidatos presidenciales 2005: así los mostramos, así los miraron", en Cuadernos de Información, $\mathrm{n}^{\mathrm{o}} 19$, pp. 89-96.

VAN ACKER, Elizabeth (2003): "Media representations of women politicians in Australia and New Zealand: High expectations, hostility or stardom", en Policy \& Society, vol. 22, $\mathrm{n}^{\mathrm{o}}$ 1, pp. 116-136.

VAN ZOONEN, Liesbet (2006): "The personal, the political and the popular: A woman's guide to celebrity politics", en European Journal of Cultural Studies, vol. $9, \mathrm{n}^{\circ} 3$, pp. 287-301. 